\title{
Treatment of cardiac sarcoidosis: a comparative study of steroids and steroids plus
}

\section{immunosuppressive drugs}

Thomas Ballul ${ }^{\mathrm{a}}$, Raphael Borie ${ }^{\mathrm{b}, \mathrm{c}}$, Bruno Crestani ${ }^{\mathrm{b}, \mathrm{c}}$, Eric Daugas ${ }^{\mathrm{d}, \mathrm{c}}$, Vincent Descamps ${ }^{\mathrm{e}}$, Philippe Dieude ${ }^{\mathrm{f}, \mathrm{c}}$, Antoine Dossier ${ }^{\mathrm{a}}$, Fabrice Extramiana ${ }^{\mathrm{g}}$, Damien van Gysel ${ }^{\mathrm{h}}$, Thomas Papo $^{\mathrm{a}, \mathrm{c}, \mathrm{i}}$, Karim Sacre ${ }^{\mathrm{a}, \mathrm{c}, \mathrm{i}}$

a-Département de Médecine Interne, Hôpital Bichat, Université Paris Diderot, PRES Sorbonne Paris Cité, Assistance Publique Hôpitaux de Paris, Paris, France

b-Département de Pneumologie, Hôpital Bichat, Université Paris Diderot, PRES Sorbonne Paris Cité, Assistance Publique Hôpitaux de Paris, Paris, France

c-Département Hospitalo-Universitaire FIRE (Fibrosis, Inflammation and Remodelling in Renal and Respiratory Diseases), Paris, France

d-Département de Nephrologie, Hôpital Bichat, Université Paris Diderot, PRES Sorbonne Paris Cité, Assistance Publique Hôpitaux de Paris, Paris, France

e-Département de Dermatologie, Hôpital Bichat, Université Paris Diderot, PRES Sorbonne Paris Cité, Assistance Publique Hôpitaux de Paris, Paris, France

f-Département de Rhumatologie, Hôpital Bichat, Université Paris Diderot, PRES Sorbonne Paris Cité, Assistance Publique Hôpitaux de Paris, Paris, France

g-Département de Cardiologie, Hôpital Bichat, Université Paris Diderot, PRES Sorbonne Paris Cité, Assistance Publique Hôpitaux de Paris, Paris, France

h-Département d'Information Médicale, Hôpital Bichat, Université Paris Diderot, PRES Sorbonne Paris Cité, Assistance Publique Hôpitaux de Paris, Paris, France i-INSERM U1149, Paris, France

All authors take responsibility for all aspects of the reliability and freedom from bias of the data presented and their discussed interpretation 


\section{Corresponding author:}

Professor Karim Sacre, MD, PhD

Department of Internal Medicine, Bichat Hospital, APHP

46 rue Henri Huchard, 75018, Paris, France

Phone : 33140256019 Fax : 33140258845 karim.sacre@aphp.fr

\section{Conflicts of interest: none}

\section{Funding: none}




\section{ABSTRACT}

Background: We aimed to compare the efficacy of steroids alone or associated with immunosuppressive drugs for the prevention of relapse in cardiac sarcoidosis (CS).

Methods: In this monocentric multidisciplinary retrospective single center study, all consecutive patients with histologically proven sarcoidosis hospitalized from January 2012 to December 2016 were considered. All patients with symptomatic CS were studied. Patients received steroids or steroids plus immunosuppressive drugs (IS) for CS treatment at diagnosis. The efficacy of each treatment strategy (steroids vs steroids + IS) was assessed by the cardiac relapse rate over follow up.

Results 326 consecutive patients with histologically proven sarcoidosis were screened. Among them, 36 (11\%) had symptomatic CS (20 (55.5\%) men, median age at diagnosis 48.5 [22.8-76]). Twenty-four patients received steroids and 12 received steroids + IS (azathioprine $n=5$, methotrexate $n=5$, cyclophosphamide $n=2$ ) at CS diagnosis. Over a median follow up of 3.6 [1-15.2] years, $13(36.1 \%)$ patients suffered a cardiac relapse including reduced left ventricular ejection fraction (LVEF, $n=4)$, third degree heart block $(n=2)$, atrio-ventricular $(n=1)$ or ventricular $(n=1)$ tachycardia and sudden cardiac death $(n=1)$. Except for a higher frequency of black patients in patients receiving IS, CS features at diagnosis and median time to relapse did not significantly differ between patients who did or did not receive IS. Relapse rate was $45.8 \%$ in the steroids group versus $16.7 \%$ in the steroids+IS group ( $\mathrm{p}=0.048)$.

Conclusions: In cardiac sarcoidosis, the combination of steroids with immunosuppressive drugs might reduce the risk of cardiac relapse, as compared to steroids alone.

Key Words: cardiac sarcoidosis; immunosuppressive drugs; treatment; relapse 


\section{INTRODUCTION}

Sarcoidosis is a multisystem granulomatous disease of unknown cause characterized by noncaseating granuloma ${ }^{1}$. Symptomatic cardiac features are reported in 5-10\% of patients with sarcoidosis, with a wide range of clinical manifestations, including congestive heart failure, conduction block or cardiac arrhythmias and heart involvement is the leading cause of death in sarcoidosis ${ }^{2-5}$. Corticosteroids therapy, although untested in randomized clinical trial, is the mainstay of treatment for cardiac sarcoidosis (CS) ${ }^{6,7}$. Immunosuppressive drugs, such as methotrexate, azathioprine or cyclophosphamide, have also been used in the treatment of severe sarcoidosis, but specific data in CS are limited ${ }^{8-11}$. We conducted a single center collaborative retrospective study to compare the efficacy of steroids alone or combined with immunosuppressive drugs in preventing CS relapses.

\section{METHODS (see supplementary materials for details)}

\subsection{Patients}

All consecutive adults patients hospitalized over a 5-years period with histologically proven sarcoidosis from our tertiary referral university hospital were retrospectively reviewed. Patients with symptomatic heart involvement were included using CS definition according to

the Heart Rhythm Society (HRS) criteria ${ }^{12}$. Cardiac sarcoidosis relapse was defined by the onset of a new CS manifestation. The local ethics committee approved the study (Institutional Review Board IRB 00006477 of HUPNVS, Paris 7 University, AP-HP).

\subsection{Data collection}

International Classification of Disease code (ICD-10) for sarcoidosis (D86) was used for screening. 


\subsection{Statistical analysis}

The primary outcome feature was cardiac relapse rate from the CS diagnosis over follow up. Comparisons between patients with and without cardiac sarcoidosis relapse and between patients who did or did not receive immunosuppressive drugs (IS) were made using MannWhitney tests for continuous variables and $\mathrm{Chi}^{2}$ or Fischer exact tests for categorical variables. Kaplan-Meïer method was used to represent cardiac relapse according to the treatment (i.e. with or without IS) initiated at cardiac sarcoidosis diagnosis.

\section{RESULTS}

\subsection{Patients' characteristics at cardiac sarcoidosis diagnosis}

From January 2012 to December 2016, 326 consecutive patients were admitted with histologically proven sarcoidosis. Among them, 36 (11\%) suffered from symptomatic cardiac sarcoidosis (Figure S1). Twenty patients $(55.5 \%)$ were male and $26(72.2 \%)$ were black. The median age at sarcoidosis diagnosis was 48.5 [22.8-76]. The delay between sarcoidosis diagnosis and heart involvement was $1.6[0-32.2]$ years with $17(47.2 \%)$ patients displaying a cardiac manifestation of sarcoidosis at sarcoidosis diagnosis. In addition to cardiac involvement, patients had a median of 2 [1-6] organs affected by the disease, including lung in all cases. Eight $(22.2 \%)$ patients were already receiving steroids (prednisone, 14 [5-30] $\mathrm{mg} / \mathrm{d})$ at CS diagnosis.

\subsection{Cardiac sarcoidosis}

The symptoms and signs that revealed cardiac sarcoidosis were unexplained dyspnea $(n=2$, $5.5 \%)$, palpitation $(n=5,13.9 \%)$, syncope $(n=5,13.9 \%)$, chest pain $(n=13,36.1 \%)$ and/or abnormal EKG $(n=26,72.2 \%)$ consistent with atrial/ventricular tachycardia $(n=9,25 \%)$, 
advanced atrioventricular block $(n=12,33.3 \%)$ or $\mathrm{LVEF}<50 \%(\mathrm{n}=13,38.9 \%)$ identified after further investigations.

At CS diagnosis, all patients received high dose steroids (prednisone, 60[20-100] mg/d) alone $(n=24)$ or associated with immunosuppressive drugs (IS) $(n=12)$ including oral azathioprine $(n=5)$, oral methotrexate $(n=5)$ or intravenous cyclophosphamide $(n=2)$. Azathioprine was given at $2 \mathrm{mg} / \mathrm{kg} /$ day. Methotrexate was given at 15 to $20 \mathrm{mg} /$ week. Cyclophosphamide was given at $0.7 \mathrm{mg} / \mathrm{m}^{2}$ every 4 weeks for 24 weeks. Methylprednisone intravenous pulses were administered in $9(25 \%)$ cases. Pacemaker or implantable cardioverter defibrillator (ICD) insertion was required in $8(22.2 \%)$ and $5(13.9 \%)$ patients, respectively. Characteristics of patients are listed in Table 1.

\subsection{Outcome}

Over a median follow up of 3.6 [1-15.2] years, 13 (36.1\%) patients suffered CS relapse. All patients were still receiving specific treatment (steroids alone or steroids and IS for 1.5 [0.56.8] years) at time of relapse. Relapse were severe in most cases $(69.2 \%, 9 / 13)$ including reduced LVEF $(n=4)$, third degree heart block $(n=2)$, sustained atrio-ventricular $(n=1)$ or ventricular $(n=1)$ tachycardia and sudden cardiac death $(n=1)$. In 4 cases, cardiac relapse was defined by the occurrence of left ventricular dyskinesia associated with myocardial FDG uptake on cardiac PET.

Interestingly patients who had cardiac relapse were more frequently male $(\mathrm{p}=0.052)$, less frequently black $(\mathrm{p}=0.008)$ and less frequently treated with immunosuppressive drugs $(\mathrm{p}=0.085)$ as compared with patients who did not relapse (Table S1). In the same line, the frequency of cardiac relapse was lower in patients treated with steroids and IS at cardiac sarcoidosis diagnosis $(16.7 \%)$ than in patients treated with steroids alone $(45.8 \% ; \mathrm{p}=0.048)$ (Table 1). Among the 9 patients who experienced severe cardiac relapse, 7 (77.8\%) were 
treated by steroids alone. Although the frequency of black patients was higher in patients receiving IS ( $\mathrm{p}=0.014)$, CS features at diagnosis did not differ between patients who did or did not receive IS (Table 1). In Kaplan-Meier analysis, treatment with steroids alone tended to be associated with a higher risk of cardiac relapse (HR 2.961, 95\% CI 0.66-13.48; log-rank $\mathrm{p}=0.141)($ Figure 1).

In patients receiving steroids alone ( $\mathrm{n}=11 / 13,84.6 \%$ of cardiac relapses), treatment of relapse associated increased of prednisone daily dose and initiation of IS in all cases including cyclophosphamide $(n=5)$, methotrexate $(n=4)$, azathioprine $(n=1)$ and mycophenolate mofetil $(n=1)$. In patients receiving steroids and IS $(n=2 / 13,15.4 \%)$ at time of cardiac relapse, treatment was modified by increasing prednisone daily dose and switch (mycophenolate mofetil instead of methotrexate) IS. In addition, pacemaker and ICD were inserted in 1 and 3 patients at cardiac relapse, respectively. At last visit, for a median follow up of 3.6 [1-15.2] years, patients had received 3.1 [1-12.8] years of steroids overall. Thirty $(88.2 \%)$ and 21 (58.3\%) patients were still treated by steroids and IS, respectively.

\subsection{Adverse events}

Severe infection occurred in $6(16.7 \%)$ patients: $4(16.7 \%)$ in patients receiving steroids alone and $2(16.7 \%)$ in patients receiving steroids and IS. Infections were mainly respiratory (undocumented bronchitis and pneumonia) and no opportunistic infections were reported. Four $(16.7 \%)$ patients receiving steroids alone and $3(25 \%)$ receiving steroids and IS patients developed diabetes mellitus. One patient (steroids and IS) developed cardiovascular events. No patients had cancers during follow up. Two (8.3\%) patients receiving steroids alone died: 1 from severe influenza $(n=1)$ and 1 from sudden cardiac death related to CS relapse $(n=1)$. One $(8.3 \%)$ patient receiving steroids and IS died from severe undocumented infection. 


\section{DISCUSSION}

Our single-center study of sarcoidosis patients with symptomatic heart involvement shows that 1-the frequency of cardiac relapse overtime is high, 2- cardiac relapse may be lifethreatening and 3- the association of steroids and IS started at CS diagnosis may have a better efficacy on cardiac relapse than steroids alone. To our best knowledge, no other study has addressed specifically the impact of adding immunosuppressive drugs to high-dose steroids on CS outcome.

Symptomatic heart involvement has been reported in 5 to $10 \%$ of patients, consistent with the $11 \%$ prevalence observed in our 326 consecutive patients with biopsy-proven sarcoidosis. Differences in prevalence and severity of sarcoidosis have been linked to age, sex and ethnic origin ${ }^{1,4}$. In our series CS patients were mostly male and black. While cardiac sarcoidosis manifestations range from asymptomatic electrocardiographic changes to sudden death, our series included only symptomatic CS patients. Although isolated cardiac sarcoidosis has been reported ${ }^{13}$, all patients in our study had evidence of extra-cardiac disease and almost half had cardiac involvement at sarcoidosis diagnosis.

The frequency of symptomatic cardiac relapse was high (36.1\%) and consistent with previous reports ${ }^{14,15}$. Randomized trials are lacking and no consensus exists among experts regarding the treatment of $\mathrm{CS}^{16}$. Although evidence-based data are poor, steroid therapy is considered mandatory ${ }^{6,7,15,17}$. Immunosuppressive agents are usually considered for steroid-resistant cases or as a steroid-sparing strategy ${ }^{18}$. A prospective study of 17 patients with a 5 years follow up reported benefit from the association of steroids and low dose methotrexate on CS relapse defined on subclinical parameters ${ }^{19}$. More recently, a single-center cohort of 73 patients identified the use of mycophenolate mofetil treatment as associated with improved CS survival on univariate analysis ${ }^{20}$. Our comparative study is the first to report on the 
benefit of high-dose steroids and IS combination started at diagnosis for clinically apparent CS.

Our study has several limitations. Its retrospective scheme from a single center and the small sample size limit the statistical power and impede the ability to generalize the findings. Since CS is rare, proper randomized trials are however exceedingly difficult to mount. Although the main clinical characteristics of the 2 groups of patients were not significantly different (Table 1), IS may have been added to steroids in patients with more severe disease, as suggested by a higher frequency of black patients and patients with reduced LVEF both known to be

associated with impaired outcome ${ }^{1,2,6,14,20}$. With such a bias, a worse outcome would have been expected in the more severe patients treated with IS. In contrast, a lower relapse rate was significantly associated with combined steroids and IS treatment. Because of the small number of patients, no specific IS drugs (azathioprine vs methotrexate vs cyclophosphamide) efficacy analysis could be performed.

In conclusion, our preliminary study suggests that the combination of high-dose steroids and immunosuppressive drugs at diagnosis is more effective than steroids alone in preventing relapse in symptomatic cardiac sarcoidosis.

\section{ACKNOWLEDGEMENTS}

KS had full access to all of the data in the study and takes responsibility for the integrity of the data and the accuracy of the data analysis. TB, RB, BC, ED, VD, PD, AD, FE, DV, TP and KS contributed substantially to the study design, data analysis and interpretation, and the writing of the manuscript. This research received no specific grant from any funding agency in the public, commercial, or not-for-profit sectors. We are thankful to Drs J.F. Alexandra, M.P. Chauveheid, and T. Goulenok for their help with patients' screening. 


\section{CONFLICTS OF INTEREST}

The authors report no relationships that could be construed as a conflict of interest.

\section{REFERENCES}

1. Valeyre D, Prasse A, Nunes H, Uzunhan Y, Brillet PY, Muller-Quernheim J. Sarcoidosis. Lancet. 2014;383(9923):1155-1167.

2. Kandolin R, Lehtonen J, Airaksinen J, et al. Cardiac sarcoidosis: epidemiology, characteristics, and outcome over 25 years in a nationwide study. Circulation. 2015;131(7):624-632.

3. Lynch JP, 3rd, Hwang J, Bradfield J, Fishbein M, Shivkumar K, Tung R. Cardiac involvement in sarcoidosis: evolving concepts in diagnosis and treatment. Semin Respir Crit Care Med. 2014;35(3):372-390.

4. Iwai K, Sekiguti M, Hosoda Y, et al. Racial difference in cardiac sarcoidosis incidence observed at autopsy. Sarcoidosis. 1994;11(1):26-31.

5. Hunninghake GW, Costabel U, Ando M, et al. ATS/ERS/WASOG statement on sarcoidosis. American Thoracic Society/European Respiratory Society/World Association of Sarcoidosis and other Granulomatous Disorders. Sarcoidosis Vasc Diffuse Lung Dis. 1999;16(2):149-173.

6. Yazaki Y, Isobe M, Hiroe M, et al. Prognostic determinants of long-term survival in Japanese patients with cardiac sarcoidosis treated with prednisone. Am J Cardiol. 2001;88(9):1006-1010.

7. Chiu CZ, Nakatani S, Zhang G, et al. Prevention of left ventricular remodeling by long-term corticosteroid therapy in patients with cardiac sarcoidosis. Am J Cardiol. 2005;95(1):143-146. 
8. Muller-Quernheim J, Kienast K, Held M, Pfeifer S, Costabel U. Treatment of chronic sarcoidosis with an azathioprine/prednisolone regimen. Eur Respir J. 1999;14(5):1117-1122.

9. Demeter SL. Myocardial sarcoidosis unresponsive to steroids. Treatment with cyclophosphamide. Chest. 1988;94(1):202-203.

10. Baughman RP, Ohmichi M, Lower EE. Combination therapy for sarcoidosis. Sarcoidosis Vasc Diffuse Lung Dis. 2001;18(2):133-137.

11. Kim JS, Judson MA, Donnino R, et al. Cardiac sarcoidosis. Am Heart J. 2009;157(1):9-21.

12. Birnie DH, Sauer WH, Bogun F, et al. HRS expert consensus statement on the diagnosis and management of arrhythmias associated with cardiac sarcoidosis. Heart Rhythm. 2014;11(7):1305-1323.

13. Isobe M, Tezuka D. Isolated cardiac sarcoidosis: clinical characteristics, diagnosis and treatment. Int J Cardiol. 2015;182:132-140.

14. Chapelon-Abric C, Sene D, Saadoun D, et al. Cardiac sarcoidosis: Diagnosis, therapeutic management and prognostic factors. Arch Cardiovasc Dis. 2017;110(89):456-465.

15. Chapelon-Abric C, de Zuttere D, Duhaut P, et al. Cardiac sarcoidosis: a retrospective study of 41 cases. Medicine (Baltimore). 2004;83(6):315-334.

16. Hamzeh NY, Wamboldt FS, Weinberger HD. Management of cardiac sarcoidosis in the United States: a Delphi study. Chest. 2012;141(1):154-162.

17. Sadek MM, Yung D, Birnie DH, Beanlands RS, Nery PB. Corticosteroid therapy for cardiac sarcoidosis: a systematic review. Can J Cardiol. 2013;29(9):1034-1041. 
18. Sayah DM, Bradfield JS, Moriarty JM, Belperio JA, Lynch JP, 3rd. Cardiac Involvement in Sarcoidosis: Evolving Concepts in Diagnosis and Treatment. Semin Respir Crit Care Med. 2017;38(4):477-498.

19. Nagai S, Yokomatsu T, Tanizawa K, et al. Treatment with methotrexate and low-dose corticosteroids in sarcoidosis patients with cardiac lesions. Intern Med. 2014;53(5):427-433.

20. Zhou Y, Lower EE, Li HP, Costea A, Attari M, Baughman RP. Cardiac Sarcoidosis: The Impact of Age and Implanted Devices on Survival. Chest. 2017;151(1):139-148. 


\section{TABLES}

Table 1 Baseline characteristics, relapse and follow up of patients according to cardiac sarcoidosis treatment

\begin{tabular}{|c|c|c|c|c|}
\hline & $\begin{array}{c}\text { All } \\
(\mathrm{n}=36)\end{array}$ & $\begin{array}{l}\text { Steroids } \\
(\mathrm{n}=24)\end{array}$ & $\begin{array}{l}\text { Steroids + IS } \\
\quad(n=12)\end{array}$ & $\mathrm{P}$ \\
\hline \multicolumn{5}{|l|}{ Baseline } \\
\hline Age at diagnosis of sarcoidosis, year & $48.5[22.8-76]$ & $46[22.8-66.3]$ & $50.6[27.2-76]$ & ns \\
\hline Male, n (\%) & $20(55.5)$ & $14(58.3)$ & $6(50)$ & ns \\
\hline Black, n (\%) & $26(72.2)$ & $14(58.3)$ & $12(100)$ & 0.014 \\
\hline \multicolumn{5}{|l|}{ Organ involvement, $\mathrm{n}(\%)$} \\
\hline Lungs & $36(100)$ & $24(100)$ & $12(100)$ & ns \\
\hline Skin & $12(33.3)$ & $8(33.3)$ & $4(33.3)$ & ns \\
\hline Ear, nose, and throat & $11(30.6)$ & $6(25)$ & $5(41.7)$ & ns \\
\hline Eyes & $7(19.4)$ & $5(20.8)$ & $2(16.7)$ & ns \\
\hline Liver & $6(16.7)$ & $4(16.7)$ & $2(16.7)$ & ns \\
\hline Brain & $4(11.1)$ & $2(8.3)$ & $2(16.7)$ & ns \\
\hline Kidney & $2(5.6)$ & $2(8.3)$ & 0 & ns \\
\hline \multicolumn{5}{|l|}{ Cardiac Involvement, n (\%) } \\
\hline Sustained AT/VT & $9(25)$ & $5(20.8)$ & $4(33.3)$ & ns \\
\hline Second/third degree heart block & $12(33.3)$ & $9(37.5)$ & $3(25)$ & ns \\
\hline Reduced LVEF $(<50 \%)$ & $13(38.9)$ & $7(29.2)$ & $6(50)$ & ns \\
\hline Hypertrophic cardiomyopathy & $7(19.4)$ & $5(20.8)$ & $2(16.7)$ & ns \\
\hline Late gadolinium enhancement on CMR & 24/34 (70.6) & $15 / 22(68.2)$ & $9(75)$ & ns \\
\hline Myocardial FDG uptake on cardiac PET & $16 / 25(64)$ & $10 / 17(58.9)$ & $6 / 8(75)$ & ns \\
\hline \multicolumn{5}{|l|}{ Relapse } \\
\hline Delay to first relapse, year & $1.5[0.5-6.8]$ & $1.5[0.5-6.8]$ & $1.5[1-3.2]$ & ns \\
\hline Cardiac relapse, n $(\%)$ & $13(33.3)$ & $11(45.8)$ & $2(16.7)$ & 0.048 \\
\hline \multicolumn{5}{|l|}{ Follow up } \\
\hline Length of follow up, year & $3.6[1-15.2]$ & $4[1-12.9]$ & $3.4[1-15.2]$ & ns \\
\hline Length of steroids treatment, year & $3.1[1-12.8]$ & $2.6[1-12]$ & $3[1-12.8]$ & ns \\
\hline Steroids at last follow up, n (\%) & $30(88.2)$ & $21(87.5)$ & $9(75)$ & ns \\
\hline IS at last follow up, n (\%) & $21(58.3)$ & $11(45.8)$ & $10(83.3)$ & $\mathrm{ns}$ \\
\hline Severe infection, $\mathrm{n}(\%)$ & $6(16.7)$ & $4(16.7)$ & $2(16.7)$ & ns \\
\hline Death, n (\%) & $3(8.3)$ & $2(8.3)$ & $1(8.3)$ & ns \\
\hline
\end{tabular}

AT/VT, atrial tachycardia /ventricular tachycardia; LVEF, left ventricular ejection fraction;

CMR, cardiovascular magnetic resonance imaging; FDG, Fludeoxyglucose; PET, positronemission tomography; IS, immunosuppressive drugs including azathioprine $(\mathrm{n}=5)$, 
methotrexate $(n=5)$ or cyclophosphamide $(n=2)$. Severe infection defined as infection requiring IV antibiotics and hospitalization.

\section{FIGURE LEGENDS}

\section{Figure 1 Kaplan-Meier Curves of Study Population}

Patients treated with steroids + IS (CT+IS) at cardiac sarcoidosis diagnosis tended to have lower rates of cardiac sarcoidosis relapse than patients treated with steroids alone $(\mathrm{CT})$. HR = $2.961 ; 95 \%$ CI 0.66-13.48; log-rank $\mathrm{p}=0.141$ 


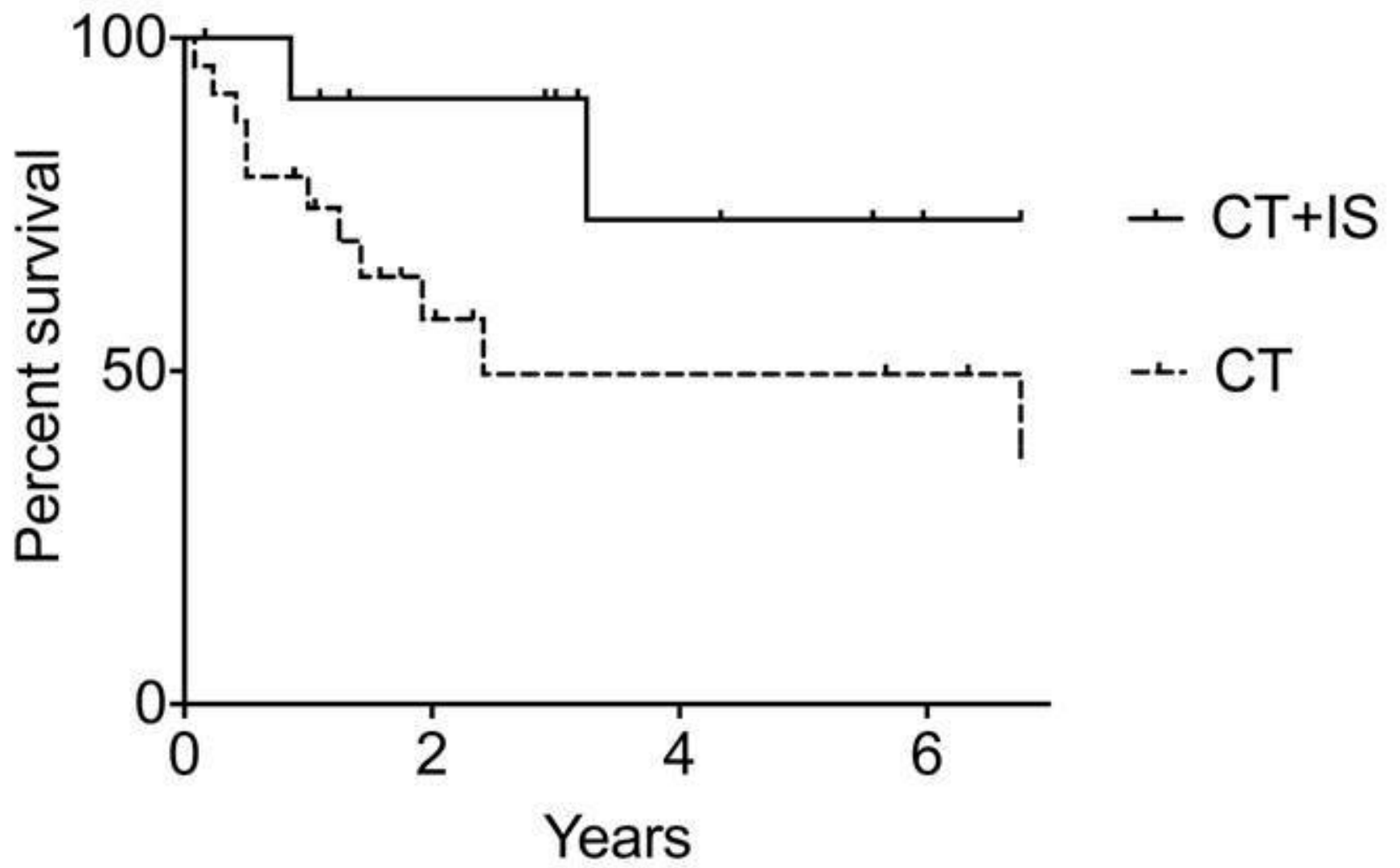

\begin{tabular}{|c|c|c|c|c|c|c|c|c|c|c|c|c|c|}
\hline & t0 & 0,5 & 1 & 1,5 & 2 & 2,5 & 3 & 3,5 & 4 & 4,5 & 5 & 5,5 & 6 \\
\hline $\mathrm{CT}$ & 24 & 19 & 16 & 12 & 9 & 6 & 6 & 6 & 6 & 6 & 6 & 6 & 5 \\
\hline $\mathrm{CT}+\mathrm{IS}$ & 11 & 10 & 8 & 7 & 7 & 7 & 5 & 3 & 3 & 3 & 3 & 3 & 1 \\
\hline
\end{tabular}

